\title{
ENSAYOS Y CARACTERIZACIÓN HIDRÁULICA DE TUBERÍAS POROSAS PARA RIEGO
}

\author{
Casado Fernández, E. ( $\left.{ }^{1}\right)(P)$, Juana Sirgado, L.( $\left(^{2}\right)$
}

1 Investigador, E.T.S. Ingenieros Agrónomos, UPM, Dpto. Ing. Agroforestal. Avda. Complutense 3, 28040 Madrid. eduardo.casado.fernandez@alumnos.upm.es

2 Profesor Titular, E.T.S. Ingenieros Agrónomos, UPM, Dpto. Ing. Agroforestal. Avda. Complutense 3, 28040 Madrid. luis.juana@upm.es

\section{Resumen.}

El presente trabajo pretende profundizar en el estudio del desagüe de las tuberías porosas, aportando ideas para su caracterización hidráulica y para la determinación de las circunstancias en las que pueden presentar un mayor potencial. Se presentan los primeros resultados experimentales con diferentes materiales y se discute sobre el funcionamiento y resultados de las mismas en posibles instalaciones. Se han realizado ensayos de caracterización de la curva de desagüe, presión-caudal, distribución de presiones y de gastos a lo largo de la tubería y se ha observado la porosidad bajo lupa binocular.

\section{Summary}

This work intends to start an in-depth study of porous pipes, delivering ideas for their hydraulic characterization, and for defining the conditions under they can be used with better results. In this paper first batch of experimental data is shown. Results have been obtained with different types of porous pipes (textile and extruded from reclaimed rubber and recycled plastic binder). Experiments have been carried out about flow-discharge features, pressureflow correspondence, and pressure/flow distribution along pipes. Porous pipes have been also observed with a stereo microscope.

\section{1) Introducción. Objetivos.}

Con el fin de poder valorar las posibilidades de las tuberías porosas de incrementar su presencia en los regadíos agrícolas actuales, se considera necesario profundizar en el funcionamiento de este sistema de riego localizado con bajo requerimientos de energía.

La información técnica suministrada por los fabricantes de los materiales de este método de riego es escasa, por comparación con la ofrecida en otros métodos, y, en algunos casos, resulta insuficiente para realizar un proyecto de riego justificado.

A diferencia con un gotero, que tiene un único punto de emisión, la multitud de puntos de emisión de las tuberías porosas le confieren ventajas e inconvenientes a su comportamiento hidráulico $\mathrm{y}$, a aspectos puntuales del mismo, como los relacionados con la obturación. En este sentido, la mayor humectación del suelo puede ser beneficiosa para algunos cultivos y también, en riego subsuperficial y en lo que se refiere a la obturación por raíces, puede presentar ventajas, pues aun llegando a penetrar las raíces en el interior, los pelos radiculares no progresan en su crecimiento tal como se ha observado con raíces de gramíneas en muestras del material extraídas de instalaciones. 
En el mercado existen básicamente dos tipos de tuberías porosas:

- las fabricadas por medio de extrusión de agregados procedentes del reciclaje de neumáticos (denominadas a continuación con el acrónimo TPE);

- las fabricadas mediante proceso textil (denominadas a continuación con TPT).

Las primeras presentan un interés adicional servir para el reciclado de un elemento problemático a nivel medioambiental. Actualmente se comercializan fundamentalmente en paisajismo y jardinería y en riegos hortícolas con pequeña superficie.

Ambos tipos de tuberías emisoras, presentan características distintas que conviene determinar mediante procedimientos experimentales, siendo necesario avanzar en la determinación de procedimientos de ensayo específicos, los cuales en un futuro, convendría normalizar de cara a tener una mayor garantía sobre sus determinaciones.

Teeluck, M. y Sutton, B.G. (1998), estudian experimentalmente la variación del gasto desaguado en una TPE, concluyendo que la tubería estudiada no posee las cualidades adecuadas como ramal de riego debido a su alto coeficiente de variación, del 0.20 a 0.35 , que además aumento con el paso del tiempo.

En un estudio más reciente, Pinto et al (2014) ensayan una TPE y concluyen que a todas las presiones ensayadas se obtuvo un resultado satisfactorio del coeficiente de variación (valores próximos a 0.05). Asimismo, observaron unas pérdidas de carga que no parecían corresponder con las obtenidas por las expresiones clásicas y una curva de gasto que no era bien representada por la expresión potencial típica y proponiendo otra más compleja, también empírica, que ajustaba mejor sus resultados experimentales.

En este trabajo, como un primer paso para el estudio de este tipo de materiales, se pretende la realización de los ensayos clásicos del riego por goteo en estas tuberías y su interpretación. En particular, se pretende:

1. Caracterización de la curva de desagüe, presión-caudal.

2. Caracterización de la distribución de presiones y de gastos a lo largo de la tubería y, en especial, caracterización de las pérdidas de carga frente al caudal entrante en relación con la longitud de tubería.

3. Observación bajo lupa binocular de la porosidad y caracterización de esta en la pared de las tuberías emisoras.

\section{2) Material y métodos.}

Se han realizado ensayos con cuatro tipos de tuberías diferentes, tres de fabricación textil y una tubería de extrusión de agregados de caucho reciclado (de distinto fabricante a las utilizadas en los trabajos referidos). Los ensayos se han llevado a cabo en el Laboratorio de Hidráulica de la Escuela Técnica Superior de Ingenieros Agrónomos de Madrid.

\section{Curva presión-caudal.}

Para determinar la curva de desagüe, presión-caudal, para cada tipo de tubería se cortaron y colocaron cuatro tramos de un metro de longitud alimentados desde una tubería con presión prácticamente constante en toda su longitud, ver imagen 1. El tramo de un metro de tubería porosa, en ocasiones se conectó en el desagüe de un gotero a su vez conectado a la tubería de alimentación se fue aumentando, en cada ensayo, con la tubería 


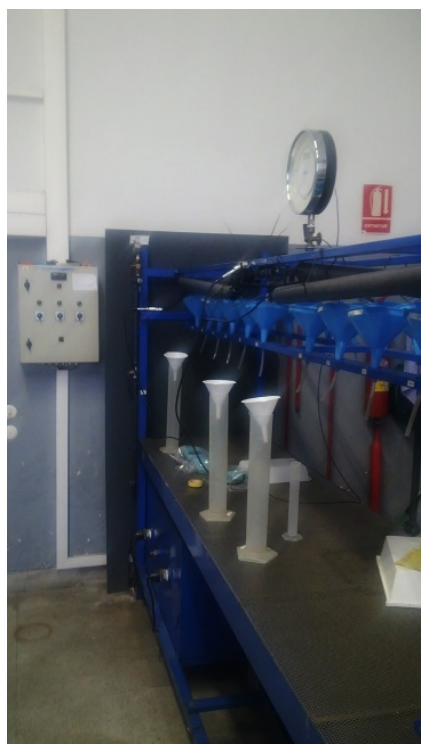

en incrementos de 5 en $5 \mathrm{kPa}$ desde cero hasta un valor máximo en función de la tubería ensayada y luego reduciéndose otra vez, con el mismo incremento, hasta llegar de nuevo a cero. La presión de alimentación se midió con manómetro de precisión con sensibilidad de $1 \mathrm{kPa}$. Adicionalmente, en un extremo de cada tubería se midió la presión con manómetro digital con una sensibilidad de $1 \mathrm{~mm}$. Para medir el gasto o volumen desaguado por unidad de tiempo se usó un cronómetro y probetas de un litro, con divisiones de $10 \mathrm{ml}$. Dado que en ocasiones se producía la emisión de chorros, la tubería se introdujo dentro de una tubería de PVC de $50 \mathrm{~mm}$ de diámetro que recogía el volumen y se colocó con una ligera inclinación para favoreces el desagüe a la probeta

Imagen.1. Banco de ensayos para obtener la curva de gasto.

\section{Pérdidas de carga y distribución de gasto a lo largo de la tubería.}

Para los ensayos de pérdida de carga y la distribución de gasto a lo largo de la tubería se extendió una longitud de entre $40 \mathrm{~m}$ hasta $100 \mathrm{~m}$, según tipo de material. El caudal se midió con un cronómetro y un contador volumétrico con una sensibilidad de $0.1 \mathrm{~L}$ y de $1.500 \mathrm{~L} / \mathrm{h}$ de caudal nominal. Las pérdidas de carga entre cabeza y cola se midieron con un manómetro digital diferencial con sensibilidad de $1 \mathrm{~mm}$ y la presión en cabeza con manómetro digital con sensibilidad e $1 \mathrm{~mm}$. El caudal a lo largo de la tubería se midió en tramos de un metro a intervalos de 10 o $20 \mathrm{~m}$, según tipo y longitud de tubería, con un procedimiento similar al comentado en la curva presión-caudal. Para cada material se realizaron varios ensayos a distintas presiones modificando la velocidad de giro de la bomba que alimenta el banco de ensayos y/o con la ayuda de llaves de paso en la misma tubería y/o en derivaciones, ver imagen 2.

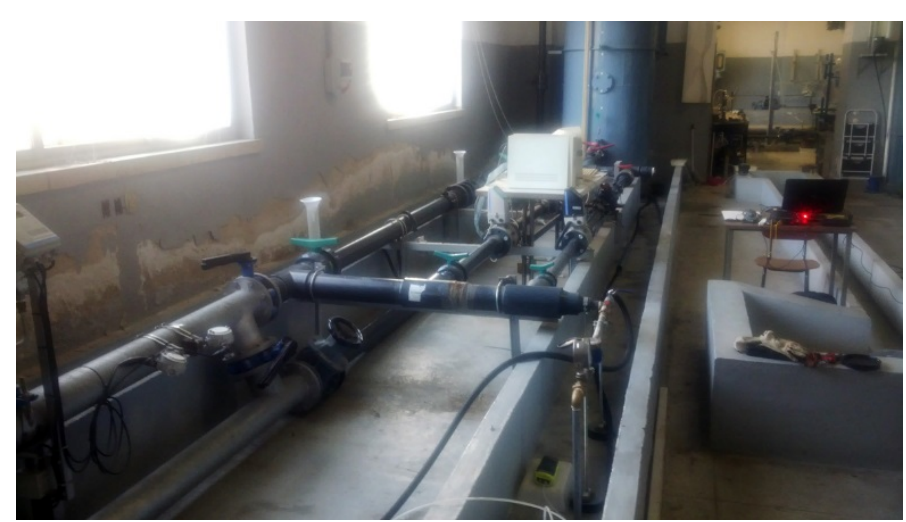

Imagen 2. Banco de ensayos para ramales.

Para obtener las imágenes de la tubería, se ha empleado una lupa binocular con un aumento 20x, equipada con una cámara de 5 megapixels. 


\section{3) Resultados.}

\section{TPT1}

El ensayo de la curva de gasto en la TPT1, mostró una importante histéresis. En la fase de caudales crecientes, a una presión próxima a $2 \mathrm{~m}$, el caudal se incrementó exponencialmente, ver figura 1 . El caudal de la fase decreciente fue considerable más alto con un exponente de la ecuación de desagüe próximo a 2.

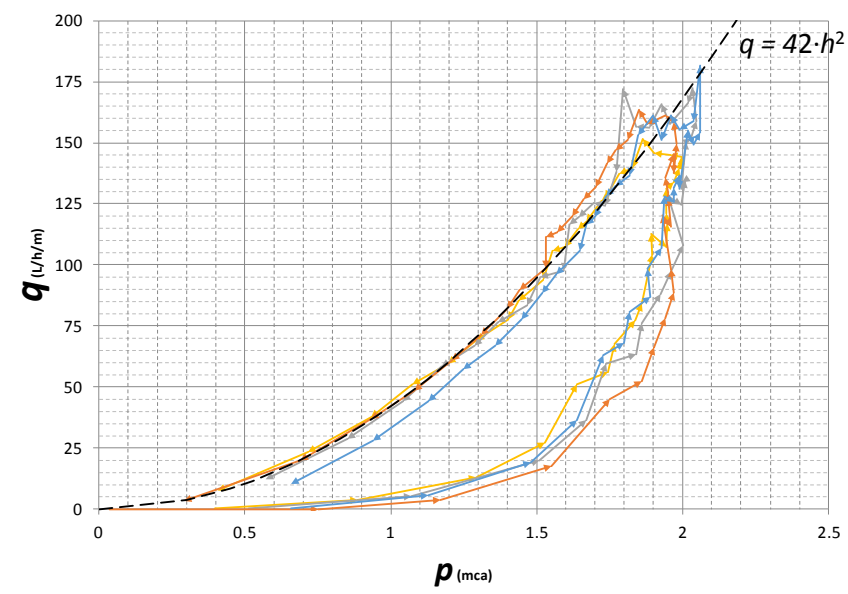

Figura 1. Curva de gasto, tubería TPT1

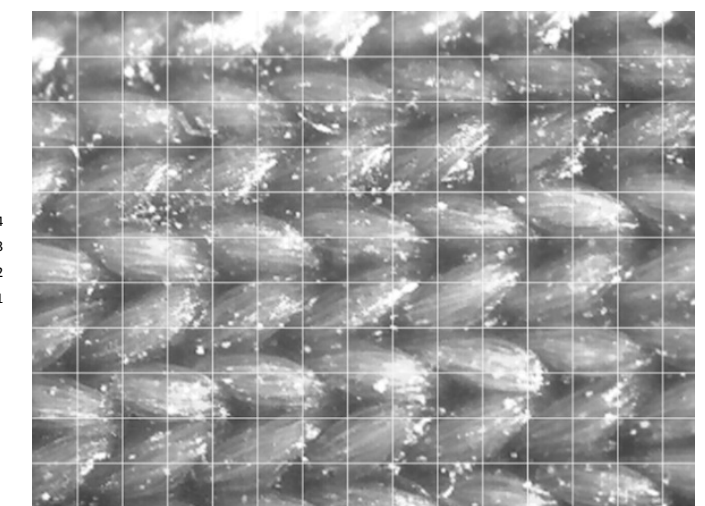

Imagen 3. Tubería porosa textil TPT1 (malla $200 \mu$ ).

La justificación del aumento exponencial de la descarga tiene que estar relacionado con el aumento de los poros que desaguan al aumentar la presión. Para que el agua empiece a salir por un poro en contacto con la atmósfera, supuesto circular, es necesario que la presión $p$ en la tubería sea mayor de $4 \sigma / d$, para vencer la sobrepresión que, por la energía superficial $\sigma$ se formaría en un menisco convexo de diámetro $d$, o dicho de otra forma, para cada presión desaguarán únicamente los poros mayores de un tamaño mínimo $d_{\min }=4 \sigma / p$. El diámetro para $p=2 \mathrm{~m}$, sería aproximadamente unas $15 \mu \mathrm{m}$ (en la imagen 4 , se muestra la pared de la tubería, en la que se observa claramente el patrón uniforme de la distribución de las fibras además de su tamaño). Cabe esperar que cuando desagüe un número importante de los poros se forme una película de agua alrededor de la tubería que elimina el menisco y, como consecuencia, desaguarían todos los poros. La fase decreciente sale un caudal mayor dado que al estar saliendo agua no debe vencerse la sobrepresión del menisco.

Si en la fase de presión decreciente desaguan la mayoría de los poros, el alto exponente de la ecuación de desagüe, $x=2$, hace pensar asimismo que la dilatación de la pared de la tubería es significativa. Las variaciones entre los cuatro ramales, aunque no es un número bajo de repeticiones, presentó valores de CV alrededor de 0.10 cuando las presiones eran mayores de $0.5 \mathrm{~m}$, siendo mayores para las presiones muy pequeñas.

Un aspecto determinante es el alto caudal desaguado lo que imposibilita proyectar grandes longitudes con el diámetro de la cinta $D=16 \mathrm{~mm}$. Sería necesario reducir el caudal hasta 20 veces para poder alcanzar longitudes de unos $50 \mathrm{~m}$ a una presión de aproximadamente $1 \mathrm{~m}$. No obstante, reducir más la presión no sería conveniente puesto que los pequeños desniveles reducirían mucho la uniformidad. 
Los ensayos en ramales y las pérdidas de carga están muy condicionados por las importantes diferencias de gasto entre el primer tramo del ramal y el último.

\section{Tubería TPT2 y TPT3}

Ambas tuberías son similares si bien la primera está pensada para trabajar a mayor presión que la segunda.

La tubería TPT2 ha presentado también algo de histéresis, ver figura 2, pero además de ser considerablemente menor, en este caso el gasto de la fase creciente, en algunos tramos, ha sido algo mayor que el de la fase decreciente. La variación del gasto con la presión también ha sido muy importante, $x \cong 2$, lo que, de aceptar el razonamiento dado en TPT1 indicaría asimismo que hay una influencia significativa de la dilatación.

El caudal desaguado es mucho más reducido que en la TPT1, la curva ajustada, tiene el mismo exponente que la representada anteriormente pero un coeficiente 54 veces menor. En la pared, imagen 5, se aprecia de una forma nítida el patrón de las fibras, y como éstas son de mayores dimensiones que las que aparecen en la Imagen 4, por lo que puede deducirse que el número de poros por unidad de superficie es menor en esta tubería.

Las desviaciones promedio relativas de los caudales observados con respecto a la curva de juste única representada han sido 0,22 , si bien, este valor se reduce a 0,14 para las presiones mayores de $2 \mathrm{~m}$ y a 0,11 para las mayores de $4 \mathrm{~m}$. Como consecuencia, si bien, los valores son más altos de lo que es frecuente en riego por goteo los resultados podrían ser aceptables si se mantienen las características.

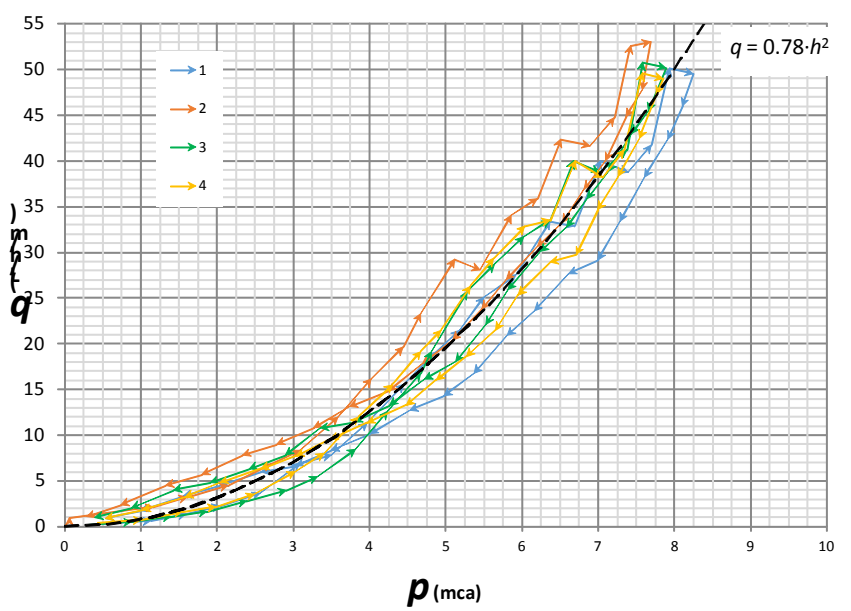

Figura 2. Curva de gasto, tubería TPT2

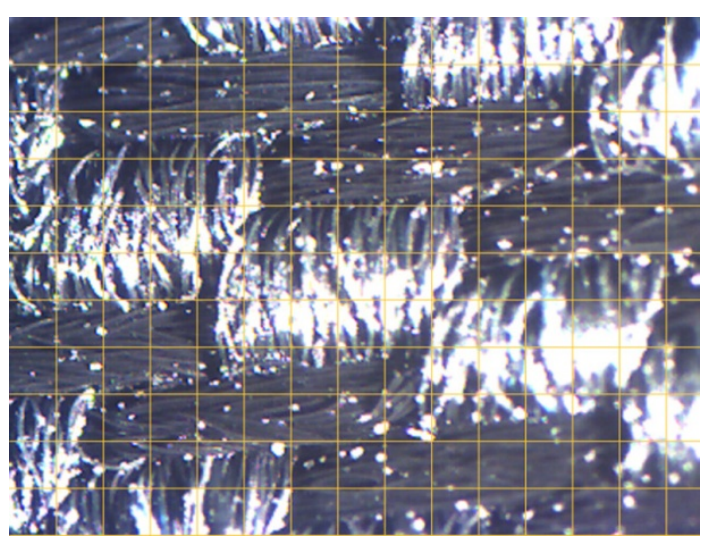

Imagen 5. Tubería porosa textil TPT2

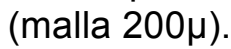




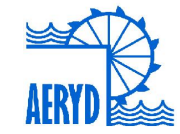

XXXIII Congreso Nacional de Riegos

Universitat Politècnica de València, Valencia 2015

DOI:http://dx.doi.org/10.4995/CNRiegos.2015.1519

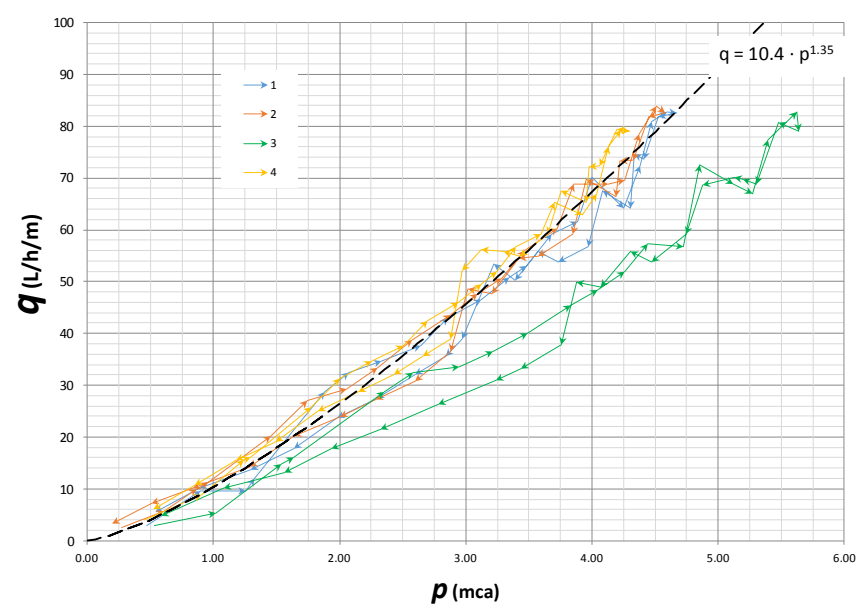

Figura 3. Curva de gasto, tubería TPT3

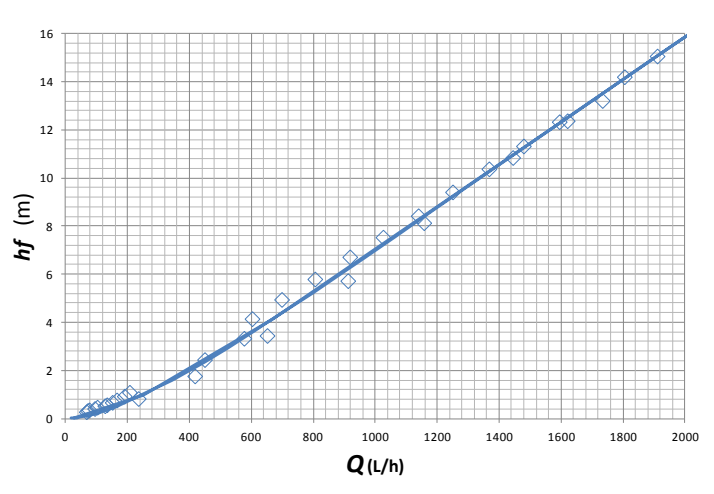

Figura 4. Curva de caudal pérdidas de carga obtenida en la tubería TPT2 y ajustada con las expresiones clásicas.

La TPT3 ha presentado también menor histéresis (ver Figura 3), pero uno de los tramos ensayados ha dado un gasto significativamente distinto a los otros tres. La variación del gasto con la presión ha sido importante pero menor que en las dos anteriores, $x \cong 1.35$, lo que, lo que indicaría una influencia de la dilatación pero menor, quizás pueda ser un resultado de haber alcanzado una menor presión máxima. Las desviaciones promedio relativas de los caudales observados con respecto a la curva de juste única pintada eliminando el tramo 3 ha sido 0,09, reduciéndose a 0,065 si se limita a las presiones mayores de $2 \mathrm{~m}$. Como consecuencia, si bien, los valores son más altos de lo que es frecuente en riego por goteo los resultados podrían ser aceptables si se mantienen las características.

Los primeros ensayos en ramales, han dado unas curvas de caudal frente a pérdidas de carga (ver Figura 4), que si bien han podido ser simuladas con el estudio iterativo clásico del ramal (las diferencias de presión han sido superiores a las que permiten usar el factor de reducción de Christiansen), ha sido necesario modificar los parámetros obtenidos previamente en la curva de gasto y usar unos factores de rozamiento muy superiores a los que cabría esperar. No obstante, también depende de los datos de las observaciones que se consideren para simular y los que se consideren para contrastar. Los resultados no se presentan con detalle pues se considera conveniente su revisión.

\section{Tubería Porosa de Extrusión (TPE)}

La TPE ha presentado un pequeño efecto de histéresis (ver Figura 5), con caudales ligeramente mayores en la fase decreciente (un coeficiente 1,91 frente a 1,81). La variación del gasto con la presión ha presentado un exponente propios del flujo en régimen laminar, $x$ $\cong 1$, que si es así el flujo por la compleja porosidad, ver imagen 6 , indicaría que no hay influencia significativa de la dilatación. 


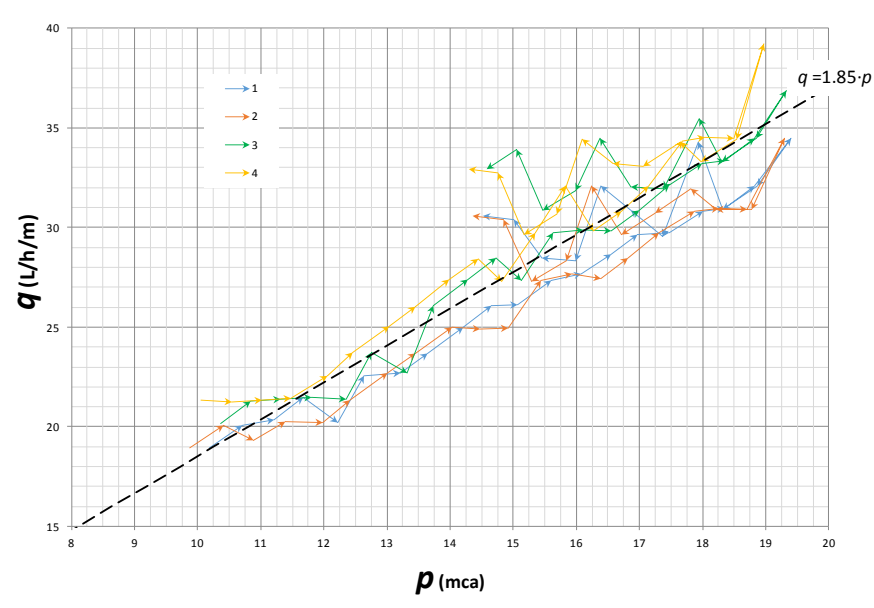

Figura 5. Curva de gasto, tubería TPT2

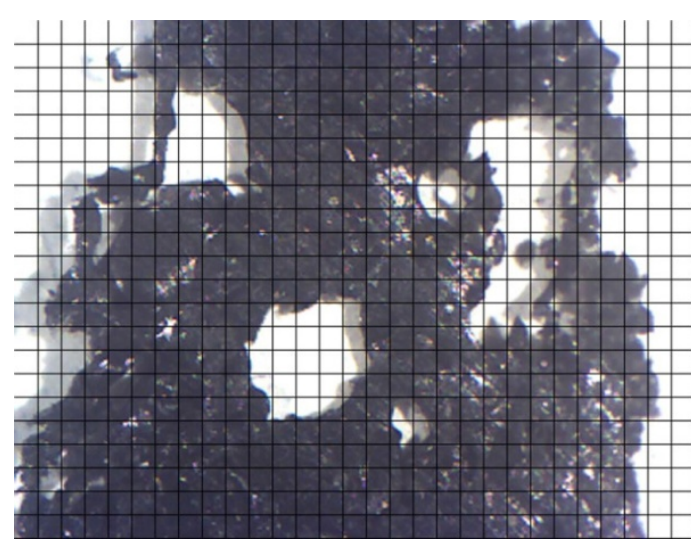

Imagen 6. Porción de una sección transversal de la tubería porosa extruida (malla 100 $\mu$ ).

Las desviaciones promedio relativas de los caudales observados con respecto a la curva de juste única pintada ha sido 0.058 lo que indicaría unas desviaciones razonables entre los cuatro tramos medidos.

En lo que se refiere a las pérdidas de carga, en este caso, el factor de reducción de Christiansen ha permitido un buen ajuste (ver Figura 6). Si bien, han presentado una aspereza importante. La expresión de Blasius, típica en ramales de riego por goteo, debido a que la tubería es muy áspera no ha resultado adecuada, siendo preferible una expresión de turbulencia completa.

En lo que se refiere a las variaciones del caudal a lo largo del ramal, las diferencias, aunque considerablemente menores, que en casos anteriores, han resultado importantes por lo que asimismo van a ser estudiadas con más precisión y, si ha lugar, se propondrá método para reducirlas. Se ha obtenido un efecto de histéresis en la descarga media del ramal de 3,5 $\mathrm{m}$ de longitud que se redujo al sumergir la tubería. Los resultados no se han introducido

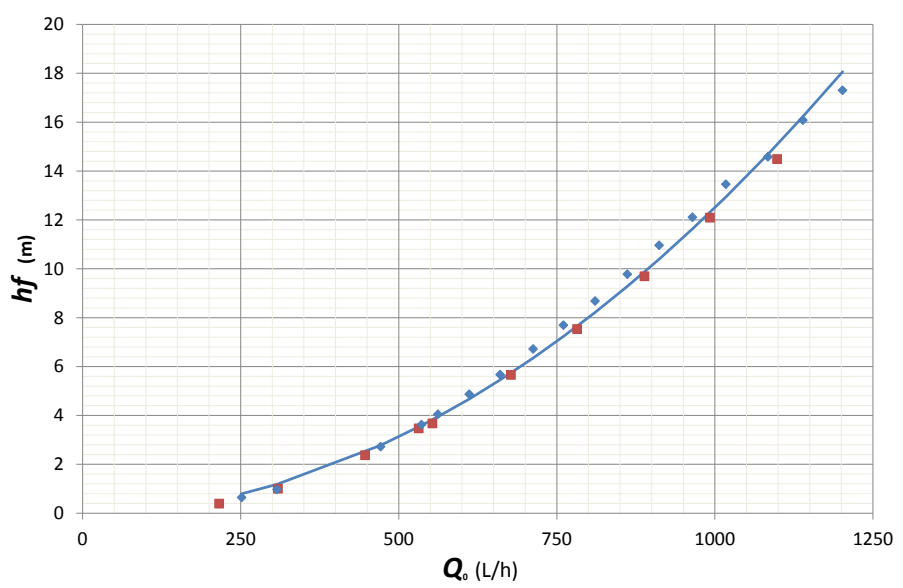

Figura 6. Pérdidas de carga en función del caudal desaguado en una tubería porosa de extrusión. Curva de ajuste con exponente 2 . considerarse necesaria su verificación y revisión

\section{Conclusiones.}

En las tuberías de fabricación textil, la ecuación de desagüe ha mostrado una gran dependencia con la presión. Son necesarios exponentes próximos a 2 e incluso mayores 
para situaciones en las que por estar seca la tubería el agua para salir del poro deba vencer la presión del menisco convexo.

El caudal desaguado es, en algunas de ellas, excesivo para poder disponer de longitudes razonables con presiones prácticas que no sean afectadas por pequeñas irregularidades del terreno. La variabilidad del desagüe achacable a la fabricación de la tubería ha presentado también valores más altos de los deseados. Si bien, el contacto de la tubería con el suelo y el cómo se humedezca la tubería, pueden tener efecto en el caudal desaguado.

Los resultados obtenidos con la fabricada por extrusión muestran una curva de gasto con menor dependencia de la presión. Por otra parte, un menor caudal desaguado para presiones moderadas, les hace potencialmente más aptas para el riego con unidades grandes. Por el contrario, en lo que se refiere a las pérdidas de carga, han presentado una aspereza importante. La expresión de Blasius, típica en ramales de riego por goteo, no ha resultado adecuada, siendo preferible una expresión de turbulencia completa.

El fenómeno de histéresis en la ecuación de desagüe, no en la de pérdidas de carga, puede estar relacionado con la curvatura de las gotas al salir, pues éste se ha reducido considerablemente sumergiendo la tubería, resultando en ese caso una curva de gasto próxima a la que se ha obtenido en la fase de descenso

Parece necesario estudiar en mayor detalle los ensayos a realizar, la forma y los parámetros a determinar y como usar luego esa información para maximizar la predicción empleando este tipo de materiales de riego.

\section{4) Bibliografía.}

Amin, M.S.M., Lim, C.W. and Zakaria, A. A. (1998). Flow Characteristics of a Porous Pipe Irrigation Lateral. Pertanika J. Sci. \& Techno!.6(1): 37 - 46 (1998)

Pinto, Marinaldo F., de Camargo, Antonio P., Neto, Osvaldo R. \& Frizzone, José A. (2014). Caracterização hidráulica de tubos porosos oriundos de pneus reciclados utilizados em irrigação subsuperficial. Revista Brasileira de Engenharia Agrícola e Ambiental v.18, n.11, p.1095-1101, 2014

Teeluck, M., Sutton, B.G. (1998). Discharge characteristics of a porous pipe microirrigation lateral. Agricultural Water Management 38 123 \pm 134

Tock, Richard Wm and A'hern, Daniel W (2003). Considerations for manufacture of irrigation pipe. Advances in Polymer Technology, Volume 2, Issue 3, pages 177-185. 